\title{
Racionalismo y retórica en Filodemo de Gadara
}

\author{
[Rationalism and Rhetoric in Philodemus of Gadara]
}

\author{
RODRIGO BRAICOVICH \\ Consejo Nacional de Investigaciones Científicas y Técnicas \\ rbraicovich@gmail.com
}

\begin{abstract}
Resumen: En este artículo analizaré algunos aspectos específicos de la obra de Filodemo de Gadara desde la perspectiva de la dimensión retórica del discurso filosófico con el fin de mostrar que el empleo que hace este pensador de ciertos dispositivos retóricos: i) no implica necesariamente un rompimiento con las normativas epicúreas respecto del uso legítimo de la retórica, ii) ni debe leerse como un abandono del proyecto racionalista epicúreo, sino que iii) puede interpretarse como expresión de una concepción sumamente rica y compleja de la racionalidad humana que incorpora las capacidades descriptivas del lenguaje como herramientas esenciales en la transmisión de la información relevante para la toma de decisiones.

Palabras clave: epicureísmo, descripción, visualización, cognitivismo, comprensión
\end{abstract}

Abstract: I aim to examine certain specific aspects of the work of Philodemus of Gadara from the point of view of the rhetorical dimension of philosophical speech in order to show that Philodemus' use of certain rhetorical devices i) does not necessarily entail a break with Epicurean norms on the correct usage of rhetoric, ii) cannot be read as an abandonment of the Epicurean rationalist project, but iii) may be interpreted as an expression of a rich and complex conception of human reason, a conception that incorporates the descriptive capacities of human language as essential tools for the transmission of relevant information in decision procedures.

Key words: Epicureanism, description, visualization, cognitivism, understanding

\section{El racionalismo epicúreo y su base cognitivista}

¿Defendía Epicuro una concepción cognitivista de la acción humana? Y, si es así, ¿̇compartieron sus discípulos esa concepción? En términos generales, parece existir un consenso algo extendido entre los especialistas en tiempos recientes en el sentido de que ambas preguntas quizá deberían recibir una respuesta afirmativa dado que las fuentes que se conservan tanto de Epicuro como de Filodemo y Lucrecio parecen evidenciar, de modo directo o indirecto, la presencia de una concepción cognitivista de la acción humana. ${ }^{1}$

${ }^{1}$ Cfr., entre otros, Annas 1989; Bobzien 2006; Nussbaum 1996; Obbink 1997, pp. 271-272; Procopé 1998; Sorabji 2000, p. 26.

Diánoia, volumen LXII, número 79 (noviembre de 2017): pp. 141-164. 
La evidencia que podría presentarse para defender la lectura cognitivista del epicureísmo es compleja: en primer lugar, ninguna de las obras y fragmentos epicúreos conservados aborda el problema del cognitivismo en forma explícita; en segundo lugar, tampoco se han conservado textos epicúreos que desarrollen en forma sistemática una teoría de la acción específica; ${ }^{2}$ por último, los pasajes que suelen presentarse como pruebas a favor del cognitivismo epicúreo proceden con frecuencia del De rerum natura de Lucrecio o del De ira de Filodemo, esto es, de obras escritas cerca de dos siglos después de la muerte de Epicuro. En suma, esto significa que la pregunta acerca de si Epicuro defendió o no una posición cognitivista de la acción humana suele responderse con base en evidencia indirecta (puesto que ninguna obra aborda el problema en forma explícita) y en buena medida recurriendo a obras alejadas en el tiempo de la fundación de las primeras comunidades epicúreas. ${ }^{3}$ ¿Es esto suficiente para cuestionar la solidez de la lectura cognitivista del epicureísmo? Creo que no, y mi razón fundamental es doble: en primer lugar, porque, por escasa que sea, la evidencia textual que aborda en forma relativamente directa el problema de la teoría de la acción humana parece favorecer con claridad la lectura cognitivista; en segundo lugar, porque la ausencia relativa de una teoría explícita de la acción se compensa ampliamente con la dimensión terapéutica que el epicureísmo otorgó tanto a la producción escrita como a las prácticas dialógicas que se desarrollaban en el seno de las distintas comunidades epicúreas. En otras palabras: aun si consideráramos que las fuentes que se conservan son insuficientes para responder la pregunta sobre el cognitivismo epicúreo, la estructura argumentativa que presentan las obras epicúreas que tenemos no posee sentido más que si suponemos que presuponen una concepción cognitivista de la acción humana.

\footnotetext{
${ }^{2}$ En palabras de J. Annas: "There does not seem to be much theory in our sources" (Annas 1989, p. 145).

${ }^{3}$ El recurso acrítico de apelar a las obras del epicureísmo italiano para cubrir las lagunas en las fuentes que se conservan de Epicuro ha sido defendido muchas veces por el carácter tan dogmático que el epicureísmo parece manifestar a lo largo de su historia y que desalentaba cualquier innovación doctrinal. No obstante, el problema no es tan sencillo, y tanto M. Erler como D. Armstrong sugieren una lectura del problema de la ortodoxia epicúrea que enfatice más bien el amplio margen de maniobra que los epicúreos italianos parecen concederse para sus propias reflexiones (cfr. Erler 1996; Erler 2011; Armstrong 2008 y Sedley 2003). El problema fundamental consiste, como señala E. Asmis, en que cuanto más innovador es un seguidor de Epicuro, más insiste respecto de su ortodoxia —que es justo lo que parece suceder en el caso de Filodemo- (cfr. Asmis 1995, p. 15).
}

Diánoia, vol. LXII, no. 79 (noviembre de 2017). 
Ahora bien, ¿en qué consiste esta concepción y qué consecuencias posee para la definición de la tarea filosófica? En pocas palabras, podríamos resumir la concepción cognitivista con la idea de que toda acción humana es el producto de la presencia en el alma del agente de la noción de que dicho curso de acción es el más conveniente o deseable. Según esto, la causa de que un individuo no alcance el telos último al que sus acciones apuntan (la eudaimonía) consiste en la presencia en su alma de opiniones erróneas - opiniones de índole muy diversa y que abarcan desde concepciones erróneas acerca del verdadero significado de ciertos fenómenos meteorológicos hasta atribuciones equivocadas de valor a ciertos bienes externos (tales como el dinero o las posesiones materiales)_. Es preciso resaltar que no es necesario que dichas opiniones hayan accedido al nivel de la conciencia plena para fungir como causas de nuestras acciones, y las razones por las que decido seguir cierto curso de acción en lugar de otro pueden ser plenamente inconscientes. ${ }^{4}$ En consecuencia, la tarea específica de la filosofía, y es aquí donde la matriz terapéutica y psicagógica del epicureísmo se revela en toda su extensión, consiste en desterrar dichas opiniones del alma del individuo ${ }^{5}$ recurriendo a medios racionales para reemplazarlas con ideas verdaderas (o, por lo menos, con ideas positivas — desde una perspectiva pragmática-).

El consenso amplio entre los especialistas en relación con la adecuación de la lectura cognitivista para el caso del epicureísmo no debe confundirse con una aceptación pasiva y acrítica: por ejemplo, D. Konstan y D. Armstrong han expresado su preocupación de que el énfasis en la dimensión cognitivista de la teoría epicúrea de la acción pueda conducir a un acercamiento peligroso con el cognitivismo estoico. Por un lado, Armstrong insiste (contra las lecturas de R. Sorabji, J. Annas, M. Nussbaum, Ph. Mitsis, J.M. Rist y T. Brennan) en el hecho de que la terapia cognitiva epicúrea no tiene por objetivo suprimir la totalidad de los estados emocionales del individuo: como señala correctamente, aun cuando el placer y la vergüenza o la ira son, en efecto, estados emocionales, ello no impide que esas emociones ocupen un lugar constructivo en el camino hacia la eudaimonía (cfr. Armstrong 2008). La crítica de Armstrong es, hasta cierto punto, demasiado general, pues al menos Mitsis y Brennan quizá rechazarían que su interpretación del epicureísmo pueda clasificarse (como hace el propio Armstrong) en térmi-

${ }^{4}$ Cfr. sobre esto Nussbaum 1996, p. 133 y Sorabji 2000, p. 27.

${ }^{5}$ Esto presupondrá, desde ya, el acto de traer a la luz las razones por las que el individuo actuó de la forma en la que lo hizo en caso de que las creencias que determinaron su acción hayan permanecido en el nivel inconsciente. 
nos de "antiemocionalista". Aun así, su advertencia merece tenerse en cuenta: es cierto que el "antiemocionalismo" y el cognitivismo son dos doctrinas conciliables, pero son lógicamente distintas, y la coincidencia de estoicos y epicúreos respecto de la segunda de ellas no implica en absoluto una coincidencia en cuanto a la primera. Que esto es así se vuelve particularmente claro, por ejemplo, cuando atendemos al De ira de Filodemo, el cual no aspira (como el De ira de Séneca) a exponer la multiplicidad de razones por las que la ira debe evitarse por todos los medios, sino más bien a señalar que existen ciertas circunstancias en las cuales la ira (la "ira natural") se encuentra plenamente justificada. Esta diferencia se debe, en pocas palabras, al hecho de que mientras que el estoicismo considera los estados emocionales esencialmente negativos, los epicúreos consideran que sólo ciertas pasiones (tales como el temor a los castigos divinos, el deseo insaciable de riquezas, etc.) deben suprimirse. ${ }^{6}$

Por su parte, D. Konstan nos ofrece una interpretación que parece alejarnos mucho de la lectura cognitivista tradicional al señalar que las dos emociones (pathe) básicas de la psicología epicúrea (a saber, el placer y el dolor) son instintivas y a-logos, porque, a diferencia de otras emociones, no implican proceso racional alguno (cfr. Konstan 2006, pp. 200-202). No obstante, cabe advertir contra Konstan que esta observación no pone en absoluto en riesgo la matriz cognitivista, pues el placer o el dolor que se experimentan ante determinado acontecimiento son un efecto de la acción y no su causa. Es cierto que el cálculo hedonístico se funda en la consideración del placer y el dolor que una decisión habrá de producir en el agente, pero es justo la consideración (racional) del placer y el dolor la que entra en juego en tal cálculo y no el placer y el dolor en cuanto sentimientos irracionales. ${ }^{7}$

Dicho sea de paso, esta aclaración resulta útil al momento de evaluar la adecuación de la propuesta de V. Tsouna de caracterizar la posición de Epicuro como un "cognitivismo moderado" (moderate o mild cognitivism; Tsouna 2007, pp. 25-27) en función del hecho de que incorpora en su análisis la dimensión puramente afectiva o no racional de la acción humana (aun de la acción virtuosa). Cabe responder a esta

\footnotetext{
${ }^{6}$ La distancia entre ambas posturas parece reducirse si incorporamos en la ecuación la concesión estoica de que existen ciertos estados emocionales positivos (eupátheiai) que pueden, en virtud de ser tales, aceptarse aun en el alma del sabio.

${ }^{7}$ Por ejemplo, E. Asmis ofreció hace poco una interpretación cognitivista sistemática de la teoría de la acción epicúrea que hace lugar en forma explícita a la dimensión estrictamente afectiva de la acción humana que subraya Konstan; $c f r$. Asmis 2011, p. 162.
} 
propuesta en términos análogos a las objeciones de Konstan, esto es, que el hecho de que cierta concepción de la acción humana sea cognitivista no implica que la conciba como absolutamente carente de elementos no cognitivos o no racionales, pues todo lo que el cognitivismo propone es que dichos elementos no poseen un papel causal primario en la acción. En otras palabras, una posición cognitivista no reduce el alma a un conjunto de proposiciones; lo que hace es afirmar que nuestras acciones y decisiones son el resultado de la interacción entre esas proposiciones - pero esa interacción causal puede acompañarse de una infinidad de elementos no racionales o tenerlos como efecto-. Es cierto que la lectura de Tsouna adquiere sentido frente al "cognitivismo radical" que supuestamente defiende Crisipo, quien en su discusión con Zenón habría propuesto considerar las pasiones no como los acontecimientos afectivos (expansiones, contracciones, etc.) que se producen en el alma como consecuencia del asentimiento a ciertos juicios erróneos, sino como los juicios mismos. Ante este panorama, aceptar la existencia de una dimensión doble en los estados emocionales (una cognitiva y otra no cognitiva) parece representar una concepción ciertamente "moderada" de una posición cognitivista. No obstante, el problema reside en que la identificación directa de las emociones con ciertos tipos de juicios es exclusiva de Crisipo, y no poseemos pruebas de que la haya defendida ningún otro estoico. Más aún: como han señalado con frecuencia los comentaristas, es muy probable que esa identificación nominal entre pasión y juicio haya representado una mera estrategia retórica de Crisipo para enfatizar el carácter necesario de la relación causal entre nuestras creencias y nuestras pasiones, y no la inexistencia de toda dimensión estrictamente afectiva en los acontecimientos anímicos. No sugiero con esto que "cognitivismo" no sea algo más que una mera etiqueta que utilizamos en forma arbitraria para clasificar una teoría de la acción; lo que intento resaltar es que la definición del cognitivismo (à la Tsouna) como una concepción que elimina por completo todo elemento no cognitivo vacía de miembros el conjunto y lo vuelve irrelevante.

Si las observaciones anteriores son correctas, el panorama que se abre ante nosotros respecto de la interpretación cognitivista del epicureísmo parece relativamente claro, al menos en cuanto al núcleo básico de dicha interpretación, a saber, la teoría epicúrea de la acción. Sin embargo, y como veremos en las secciones siguientes, el consenso sobre la adecuación de la lectura cognitivista del epicureísmo parece diluirse cuando se aborda el problema de las estrategias psicagógico-pedagógicas que sería esperable encontrar en una filosofía que se estructura alrededor de una concepción cognitivista de la acción, a saber, estrategias 
de corte racionalista. Dicho problema se vuelve más complejo en la medida en que nos alejamos de la obra de Epicuro y nos acercamos al epicureísmo italiano y, sobre todo, a la obra de Filodemo, dado que algunos aspectos de la misma han sido considerados indicios probables de un alejamiento marcado del racionalismo ortodoxo. Contra dichas lecturas, intentaré mostrar que es posible reconstruir una imagen de conjunto relativamente sistemática y coherentemente racionalista de la obra de Filodemo, y que buena parte de los elementos que han sido señalados como problemáticos en relación con dicha concepción pueden, en realidad, reinterpretarse como una proyección muy rica y compleja del proyecto racionalista original.

\section{Filodemo y las directivas epicúreas respecto de la retórica}

La posición de Epicuro ante el problema de la retórica es harto conocida y no parece haber suscitado demasiadas controversias entre sus seguidores: a diferencia del estoicismo, el cual asigna un papel paidético importante a la dimensión retórica y recurre en forma sistemática (sobre todo desde Crisipo en adelante) a las citas literarias, a las referencias a la tragedia y a los textos homéricos, Epicuro establece un ideal discursivo muy austero:

Se sirve de un estilo preciso en el tratamiento de los temas, al que el gramático Aristófanes le reprocha ser muy personal. Era tan claro, que en su obra Sobre la retórica no pretende exigir otro requisito que la claridad (saphēneia). (DL 10.13; traducción de C. García Gual)

En relación con la dimensión retórica del discurso epicúreo, H. Usener recoge unos quince testimonios que coinciden de manera invariable en este asunto: la prosa de Epicuro se destaca por ser simple, sin adornos, sin ironía. ${ }^{8}$ En palabras de Cicerón, el discurso de Epicuro es "directo, expresa conceptos simples y llanos en una forma que es fácil de entender". ${ }^{9}$ Que la prosa de Epicuro haya tenido estas características

${ }^{8}$ H.M. Hubbell sugería hace tiempo que la búsqueda de esta característica en la producción discursiva delata cierta parcialidad hacia el estilo isocrático porque la búsqueda de la claridad era justo la característica en la que se destacaba la prosa de Isócrates - algo que, dicho sea de paso, permitiría comprender por qué Filodemo asigna a Isócrates un lugar sustantivo en su tratado sobre retórica-. Cfr. Hubbell 1916, p. 418.

9 "Complectitur verbis quod vult et dicit plane quod intellegam" (Cic., Fin. 1.5.14; la traducción es mía). 
tiene, por supuesto, otras repercusiones: dada la dinámica fuertemente piramidal del epicureísmo, el estilo elegido por Epicuro es el estilo recomendado por Epicuro: no se trata sólo de que sus tratados posean ciertas características, sino de que todos los textos escritos en el ámbito de la escuela deben poseerlas. El tratado de Epicuro sobre retórica, muy crítico de la tradición retórica clásica, habría servido, en este sentido preciso, como manual de estilo para el funcionamiento interno de la escuela. ${ }^{10}$

Aunque no se impliquen mutuamente, la idea de una retórica frugal, medida, subordinada sin concesiones al objetivo de la claridad y, con ello, de la transmisión de las ideas que el epicureísmo considera el fundamento último de su propia filosofía, se articula a la perfección con la concepción cognitivista de las pasiones y de la acción humana que podemos reconstruir a partir de los textos de Epicuro, Lucrecio y Filodemo. Si partimos de esa matriz cognitivista, y si aceptamos que el objetivo último de los tratados epicúreos consiste en persuadir al lector respecto de verdad de un conjunto de doctrinas y, en consecuencia, de la necesidad de adoptar cierto estilo de vida, se hace evidente que una retórica compleja, sofisticada y erudita se vuelve por completo inesencial en el marco del proyecto pedagógico (o psicagógico) epicúreo. Al analizar el problema de la poesía en el epicureísmo, E. Asmis ha ofrecido consideraciones que parecen resumir a la perfección la íntima vinculación que es posible constatar entre la concepción epicúrea de la retórica y su proyecto pedagógico racionalista:

Como muestran con claridad los escritos de Epicuro y su círculo, el hombre sabio utiliza la prosa, y no la poesía, para ilustrar a otros. Lo que impide a Epicuro hacer de la poseía un ayudante de la filosofía es, sugiero de nuevo, su racionalismo. A pesar de que no hay duda de que Epicuro y sus seguidores practicaban adoctrinamientos irracionales, el objetivo del primero consistía en persuadir a sus alumnos apelando a sus capacidades intelectuales o, tal como él lo concebía, a través del análisis filosófico. Aspiraba a moldear su carácter por medio de opiniones no disfrazadas, las cuales debían ser aprobadas por cada persona a través de un acto mental de juicio. Con este fin en mente, exigía la claridad como la única cualidad del

${ }^{10}$ Es preciso aclarar que Epicuro posee razones importantes (si se considera la axiología epicúrea) para oponerse a la retórica clásica y a su obsesión con el adorno y la sofisticación: para el pensador de Samos, la obsesión con la forma del discurso, con la elocuencia y con las diversas prácticas declamatorias representa una desviación respecto de la verdadera tarea filosófica, la cual consiste en realizar todo lo que tengamos a nuestro acceso para alcanzar la eudaimonía, idéntico destino que parece corresponderle a buena parte de las artes liberales que conformaban la paideia tradicional ateniense. 
buen discurso. La función del discurso claro es comunicar opiniones claras y verificables por cada estudiante a través de la experiencia sensible. Los relatos falsos, la experiencia mimética, los discursos públicos, se rechazan todos como herramientas educativas porque no conducen a la persona a una visión clara de la verdad. Lo que produce una vida feliz es el "razonamiento sobrio" en todas las circunstancias; y esto depende de tener una visión clara de la naturaleza humana, impartida por el discurso claro de la filosofía. (Asmis 1995, p. 21)

Frente a este estado de cosas, la obra de Filodemo ("dialéctico sutil y pertinente, hombre de cultura destacable" según Gigante 2002, p. 24) parece ubicarnos en un horizonte radicalmente distinto por varias razones: en primer lugar, porque, en contraste con el aparente rechazo por parte de Epicuro de los estudios técnicos de retórica - junto con todas las "artes liberales" restantes-, ${ }^{11}$ Filodemo redacta un tratado específico sobre retórica que aspira a demostrar que al menos una de las ramas de la retórica (la retórica sofística o epidíctica) constituye una téchne en sentido legítimo, en la medida en que puede (a diferencia de las retóricas forense y política) producir el efecto que promete, a saber, la persuasión a través del dominio de una técnica específica de composición del discurso. ${ }^{12}$ En segundo lugar, porque de Filodemo se ha conservado no sólo parte de su producción poética (unos treinta epigramas breves), ${ }^{13}$ sino también fragmentos de su tratado técnico sobre el arte poética, ${ }^{14}$ lo cual parece contradecir de manera abierta el rechazo de Epicuro tanto de la producción poética como de la teoriza-

${ }^{11}$ Cfr., entre otros, Sexto Empírico, M I.1-5: DL X.6; Cic., Fin. I.71; Ateneo, Deipnosophistae 13.588A; Plutarco, Non posse 1094D.

${ }^{12}$ Los principales papiros a partir de los cuales se encuentra en proceso de reconstrucción el tratado sobre retórica (al parecer compuesto de diez libros) son: PHerc. 1427, 1672, 1674 (libro I); 1426, 1506 (libro III); 1007, 1423, 1673 (libro IV); 832, 1015 (libro VIII); 1004, 1669 (no asignados). Además de las ediciones parciales publicadas en las últimas décadas en Cronache Ercolanesi, la edición de conjunto de base es aún Sudhaus 1895. La traducción reciente más extensa (libros I y II) se encuentra en Chandler 2006.

${ }^{13}$ La edición crítica más reciente se encuentra en Sider 1997.

${ }^{14}$ PHerc. 994, 1676 (libro I); 1087, 1403 (libro III); 207 (libro IV); 1425, 1538 (libro V). Si bien no hay aún una edición de conjunto de la multiplicidad de fragmentos que integraban el tratado (compuesto de al menos cinco libros) De poematibus, puede leerse una excelente introducción a los diversos aspectos que conlleva la reconstrucción del texto en Janko 1995. La colección de ensayos incluidas en Obbink 1995 ofrece un panorama interesante de la complejidad del problema de la articulación de la poesía en el horizonte epicúreo. 
ción en torno a ella. ${ }^{15} \mathrm{El}$ último elemento, que es el que me interesa más en estas páginas, está representado por la dimensión retórica que puede constatarse con facilidad en la producción teórica de Filodemo y que establece una distancia decisiva con la prosa límpida, clara y libre de ornamentos (y ambigüedades) que supuestamente defiende Epicuro. En síntesis, el contraste entre la normativa epicúrea respecto de las prácticas discursivas ortodoxas y la producción que se conserva de Filodemo transita por dos sendas diferentes (aunque complementarias): la ampliación curricular y el estudio de disciplinas que tradicionalmente se consideran irrelevantes para la eudaimonía (retórica, poesía, música), por un lado, ${ }^{16}$ y la incorporación de una dimensión retórica muy elaborada en la composición de obras que no se vinculan con problemáticas retóricas o literarias (De ira; De morte; De oeconomia), por otro.

Ahora bien: ¿cómo debemos interpretar estas divergencias respecto de la normativa ortodoxa? ¿Debemos suponer que Filodemo las interpretó como tales, es decir, como desviaciones respecto de la palabra del maestro, o que, por el contrario, creyó que — por alguna razón u otrase justificaban en el horizonte mismo del epicureísmo? Más importante aún: ¿implican estas innovaciones alguna dificultad en relación con el proyecto racionalista epicúreo ${ }^{17}$

Algunos comentaristas importantes de la obra de Filodemo han sugerido en las últimas décadas que la respuesta a la pregunta anterior debe ser afirmativa, y que la obra de Filodemo representa una especie de anomalía o al menos un punto de quiebre respecto de la tradición epicúrea anterior en cuanto a las dimensiones retórica y racional del discurso filosófico. En efecto, Annas, Schroeder, Armstrong y Tsouna han defendido, con mayor o menor radicalidad, el carácter singular de la posición filodemea, ora en cuanto al interés heterodoxo de Filodemo

${ }^{15}$ Cfr. Heráclito, Alleg. Homer. 4 y 17 [Usener 229] quien, junto con Ateneo, reúne a Epicuro y a Platón en cuanto a su decisión de expulsar de la ciudad a los poetas (Deipnosphistae V.12, p. 187 C, [Usener 228]).

${ }^{16}$ Cabe subrayar que no es casual que Filodemo aparezca ante la mirada de Cicerón (claramente hostil al epicureísmo) como un hombre "doctísimo" ("optimi viri et homines doctissimi"; Cic., Fin. II. 35, 119), una expresión que quizá pensó para hacerla contrastar con la imagen de Epicuro (difundida - junto a otrospor el propio Cicerón) como un escritor que aconseja mantenerse alejado de toda formación intelectual que no contribuya en forma directa al logro del telos último.

${ }^{17}$ En Nussbaum 1996 y Cooper 2012 podemos encontrar objeciones al carácter eminentemente racionalista del proyecto epicúreo, explícito ya en la definición misma de filosofía que conserva Sexto Empírico: "una actividad que produce la

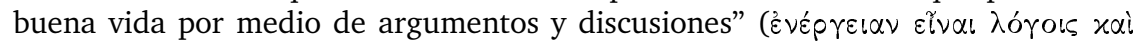

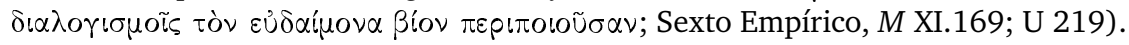


por problemáticas vinculadas de manera específica con la retórica, ora en cuanto al recurso a estrategias expositivas alejadas de una argumentación estrictamente racional.

Por ejemplo, luego de un análisis agudo de la dimensión retórica en las técnicas de visualización que aplica Filodemo en De ira y en De morte (técnicas cuya invención el autor atribuye a Filodemo y no a Lucrecio), ${ }^{18}$ Schroeder sugiere que es el recurso a dichas técnicas (que considera una innovación propia de Filodemo) el que habría permitido al epicureísmo "liberar a la filosofía de la tiranía del logos" (Schroeder 2004, p. 148). La "liberación" que menciona Schroeder consiste en específico en la incorporación en el seno de la pedagogía epicúrea de un elemento no racional, a saber, la capacidad de formar una imagen mental de una situación (por ejemplo, la muerte de un individuo o la destrucción de un pueblo), con el objetivo de que produzca en el individuo un efecto terapéutico. En una lectura análoga, Armstrong sugiere que advirtamos en el De ira una distinción clara entre argumentos (racionales) y retórica:

Podemos ver que estamos ante una cuestión de retórica y no simplemente de argumentos; que Filodemo intenta apelar a las emociones del mismo modo que a la razón. Podemos decir que su estilo ejemplifica el tipo de retórica epidíctica, la búsqueda refinada de la belleza pura en el lenguaje que, como [el propio Filodemo] argumenta en Rhetorica, constituye el arquetipo del estilo retórico. [...] En términos filosóficos, podemos decir que su estilo es protréptico, una retórica destinada a convencer a iniciados potenciales, como la poesía de su contemporáneo Lucrecio —un estilo que los epicúreos usaban sólo con precaución argumentando, como solían hacerlo, que la prosa sobria y analítica, y no la retórica elegante y menos aún la poesía, era el medio más adecuado para transmitir la verdad-. (Armstrong 2004, p. 26)

Desde esta perspectiva, retórica y paideia corren por caminos distintos: allí donde Filodemo se permite a sí mismo adornar una prosa que debería (según las indicaciones del maestro) ser clara, precisa y despojada, allí donde se permite recurrir a cierta teatralidad en la presentación de un concepto o dramatizar, mediante la creación de escenarios ficticios

${ }^{18}$ En efecto, Schroeder sugiere interpretar el uso amplio en Filodemo de las técnicas de visualización como indicio de la influencia de Filodemo en Lucrecio: "If the visualization is an original contribution of Philodemus to Epicureanism and we also find it in Lucretius, we have a good internal argument for the influence of Philodemus upon Lucretius" (Schroeder 2004, p. 149).

Diánoia, vol. LXII, no. 79 (noviembre de 2017). 
por momentos hiperbólicos, las consecuencias que se desprenden de ciertas decisiones, allí Filodemo da rienda suelta a intereses puramente estéticos y no necesarios para su tarea pedagógica.

En contraste parcial con la radicalidad de la propuesta de Armstrong, Annas y Tsouna proponen un camino apenas divergente cuando interpretan ambas dimensiones (la retórica y la argumentativa) como componentes esenciales de la tarea pedagógica. No obstante, lo decisivo es que la distinción entre ámbitos persiste: donde Armstrong vinculaba la retórica con intereses puramente estéticos, por un lado, y los argumentos racionales con los instrumentos de la paideia, Annas y Tsouna proponen vincular la retórica con las emociones y los argumentos con la racionalidad. En uno de los estudios más sólidos y sistemáticos de la ética de Filodemo, Tsouna propone, en efecto, interpretar el De libertate dicendi según ese doble eje:

Los argumentos y los elementos retóricos son condiciones necesarias y, en conjunto, componentes necesarios de la concepción de la parrhesía de Filodemo. Los primeros [los argumentos] apelan a la razón; los segundos [los elementos retóricos] agitan las emociones. (Tsouna 2007, p. 93)

En un sentido análogo, aunque en relación con el De ira, Annas sugiere tener presente una distinción tajante entre las shock tactics de la primera parte del tratado (que califica de "teatral") y la estructura expositiva de la segunda parte, estrictamente analítica (Annas 1989, p. 156).

\section{El racionalismo de Filodemo}

Las consecuencias de las interpretaciones de Schroeder, Armstrong, Tsouna y Annas respecto de la dimensión retórica del discurso de Filodemo son decisivas en la medida en que nos obligan a aceptar un abandono (parcial o total) por parte de este último del proyecto $\mathrm{ra}$ cionalista de Epicuro. Sin embargo, y como intentaré argumentar, el carácter innovador de la obra de Filodemo no radica en el hecho de que recurra, contra las directivas epicúreas, a elementos extraargumentales en la construcción del discurso, sino en que funciona con un concepto de racionalidad mucho más rico y complejo que el que subyace en la distinción (reductiva) entre racionalidad y retórica. Lo que Schroeder, Armstrong, Annas y Tsouna interpretan como la dimensión estrictamente afectiva del discurso de Filodemo puede considerarse, en pocas palabras, una dimensión puramente racional, integrable en los procesos argumentativos de la deliberación. Como intentaré demostrar en 
las secciones siguientes, este cambio de perspectiva no se reduce a una mera sustitución de etiquetas: por ejemplo, considerar las descripciones de Filodemo de la figura del iracundo como dispositivos racionales en lugar de "tácticas de choque" significa que Filodemo espera que dichas descripciones funcionen como premisas en un silogismo práctico y no como instrumentos de adoctrinamiento afectivo. ${ }^{19}$ Desde esta perspectiva, antes que un abandono del proyecto racionalista o de una liberación respecto de la "tiranía del logos", lo que ofrece la obra del de Gadara es más bien una concepción enriquecida del racionalismo, la cual, según creo, Epicuro no tendría razones para rechazar.

\subsection{Los epigramas y De poematibus}

Si bien, como señala Asmis, la dimensión racionalista de la pedagogía epicúrea hace innecesario desde un punto de vista lógico el recurso a cualquier dispositivo retórico que pueda oscurecer la "visión clara de la verdad" (Asmis 1995, p. 21), eso no significa que ese recurso sea contradictorio con el proyecto racionalista. En efecto, y como señala la propia autora, el rechazo de Epicuro de las artes miméticas se dirige sólo a la dimensión educativa que la tradición les asignaba (a través, sobre todo, del estudio y memorización de las obras de Homero), y no a dichas artes en cuanto fuentes de entretenimiento. ${ }^{20}$ Desde esta perspectiva, la producción epigramática de Filodemo puede hallar una justificación como fuente de placer tanto para el autor como para los comensales ante quienes los epigramas se recitaban: siempre y cuando no fuera una "práctica profesional", ${ }^{21}$ la elaboración y recitación de poemas po-

${ }^{19}$ No es casual que ninguno de los autores que proponen interpretar la obra de Filodemo como impregnada de estrategias persuasivas no racionales jamás ofrezca una explicación específica respecto de cómo Filodemo suponía que debían incidir dichas estrategias en el alma del discípulo o lector. Afirmar que estamos ante shock tactics (Annas) o ante dispositivos que "agitan las emociones" (Tsouna) no nos dice nada respecto de cómo funcionan en un horizonte cognitivista como el que defiende - hasta donde podemos ver- Filodemo.

${ }^{20}$ Cfr. Asmis 1995, p. 21 y López Martínez 2003, quien resalta el hecho de que el placer que produce la poesía no se reduce a su dimensión físico-acústica, sino que se trata más bien de un placer intelectual que se deriva del hecho de que la poesía, a diferencia de, por ejemplo, la música, se apoya en una "estructura significante" que se remite a la "significación de los pensamientos" (p. 124).

${ }^{21} \mathrm{G}$. Karamanolis señala al respecto que "la publicación de un solo libro de epigramas podría considerarse 'poco profesional' y, en consecuencia, acorde con la opinión de Epicuro de que el hombre sabio no debería abocarse a la poesía" (Karamanolis 2005, p. 122). Empero, cabe advertir que Karamanolis parece seguir a Asmis en su rechazo de la lectura tradicional de DL X.121 como prohibición de 
día ser una fuente de placer tan genuina como cualquier otro de los placeres considerados legítimos en el seno de la comunidad epicúrea. ${ }^{22}$ Por otra parte, puede ofrecerse una justificación análoga para el caso de la teorización de Filodemo respecto de los criterios estéticos sobre los que construye la poesía: ${ }^{23}$ en la medida en que la producción poética (en cuanto fuente eventual de entretenimiento) requiere el conocimiento de cierta téchne, el conocimiento de esta última deviene en una condición necesaria de aquélla y se halla, por lo tanto, justificada como instrumento indirecto para la consecución de cierto placer estético.

Sin embargo, la producción epigramática de Filodemo permite una segunda lectura: D. Sider ha sugerido, en efecto, que "los epigramas no son meramente consistentes con las doctrinas que encontramos en la prosa de Filodemo, sino que fueron pensados para ilustrarlas". ${ }^{24} \mathrm{Si}$ bien el autor analiza varios de los epigramas conservados, el tercero merece atención especial por un motivo adicional, que es el hecho de que articula el discurso poético no sólo con la práctica de la parrhesía, sino también con el reconocimiento por parte de Filodemo de la necesidad de tomar en cuenta el orgullo propio del individuo, que se censura por ser una barrera para el progreso moral. En efecto, dicho epigrama escenifica un diálogo entre un individuo que, en estado de congoja ante la perspectiva de su futura muerte, ruega a Janto que acompañe con su música un lamento sobre el carácter inevitable del fin de la vida humana. Poniendo en juego una franqueza sin tapujos, Janto le ofrece un verso que fuerza en el lector la comprensión profunda, decisiva, del carácter siempre inminente de la muerte. La escena del epigrama remeda las prácticas parrhesiásticas descritas por Filodemo como parte de la dinámica pedagógica de la comunidades epicúreas, a la vez que atiende sus límites mediante una táctica conocida, "criticar a otro criticándose a sí mismo" (Sider 2004, p. 94), como estrategia para sortear la barrera del amor propio del alumno, lector u oyente.

Según esta perspectiva, una buena parte de los epigramas de Filodemo puede interpretarse, si seguimos a Sider, como una dramatización muy rica y compleja, diseñada a la medida de un auditorio específico,

\footnotetext{
"poetizar" o “ejercer la actividad poética” (traducción de García Gual); tanto Asmis

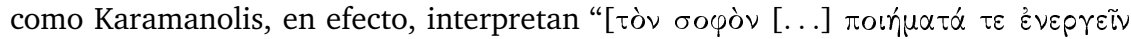

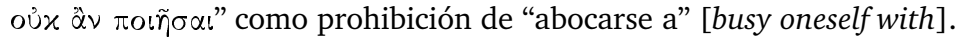

${ }^{22}$ Véanse Asmis 1995 y Armstrong 2002, pp. 308-309.

${ }^{23}$ Cfr. supra nota 14.

24 "The epigrams are not merely consistent with but are intended to illustrate doctrines found in his prose" (Sider 2004, p. 85).
} 
de ciertas doctrinas epicúreas ya expuestas en los tratados en prosa. ${ }^{25}$ Como se hace evidente, los epigramas no devienen de esta forma en una mera fuente de entretenimiento legítima por el mero hecho de no poner en riesgo el proyecto racionalista, sino que pretenden ser un instrumento de dicho proyecto. Esto parece conducir a un problema relativamente serio en la medida en que la atribución de una intencionalidad pedagógica a los epigramas parece poner en cuestión la distinción propuesta por Asmis entre las dos dimensiones posibles de la poesía, i.e., como entretenimiento y como herramienta educativa, de las cuales sólo una hallaría justificación en el sistema epicúreo. Como sugeriré en la siguiente sección, este problema se disuelve de manera parcial si atribuimos a Filodemo una concepción rica y compleja de la racionalidad que no reduzca la dinámica del logos a la comprensión de proposiciones, sino que incorpore las capacidades descriptivas del lenguaje como una herramienta esencial al momento de transmitir la información relevante para la toma de decisiones.

\subsection{De ira, De morte, De oeconomia}

$\mathrm{Al}$ menos tres de las obras que se han conservado de Filodemo, De ira, De morte y De oeconomia recurren (con mayor o menor frecuencia) a descripciones detalladas y vívidas de situaciones o personajes que se vinculan de modo directo con ciertas problemáticas filosóficas específicas. En esas descripciones en las que Filodemo despliega al máximo su habilidad retórica, se escenifican en forma muy dramática, por ejemplo, las consecuencias concretas que se siguen de adoptar ciertos cursos de acción, los efectos que ciertos rasgos del carácter de un individuo pueden producir en sus relaciones con los individuos que lo rodean o las repercusiones del hecho de guiarse por máximas equivocadas para alcanzar la eudaimonía. ${ }^{26}$

${ }^{25}$ En rigor, dicha interpretación representa una suerte de radicalización de la lectura que ofrece el propio Sider en su introducción a los epigramas de Filodemo (Sider 1997, pp. 32-39), donde reconocía el carácter pedagógico de algunos epigramas pero aceptaba, al mismo tiempo, la presencia de un número de elementos no necesariamente epicúreos en otros y, en consecuencia, proponía que si bien algunos de los epigramas podían haber tenido una función pedagógica estrictamente epicúrea, algunos de ellos parecían escritos con fines puramente estéticos - aunque claramente subordinados a los lineamientos del hedonismo epicúreo-. Sobre esto último cfr. las observaciones de Asmis a la exposición de Plutarco en Asmis 1995, pp. 19-22.

${ }^{26}$ Si prestamos atención a los vínculos entre Filodemo y Virgilio, sería interesante desarrollar un trabajo para vincular las técnicas descriptivas de Filodemo con las

Diánoia, vol. LXII, no. 79 (noviembre de 2017). 
Ahora bien, ¿cómo funcionan dichas descripciones en términos persuasivos? ¿Lo hacen en un plano distinto del de la "argumentación racional"? ¿Apelan a una dynamis distinta del alma o a una dimensión puramente emocional? Algo de ello es lo que parece subyacer en las interpretaciones de Armstrong, Schroeder, Annas y Tsouna o, cuando menos, en la distinción que todos ellos proponen, mutatis mutandis, entre la dimensión retórica (vinculada con lo afectivo) y la dimensión estrictamente argumental (vinculada con lo racional). ¿Es ésta la única lectura posible de las estrategias de visualización que emplea Filodemo? Sugiero que no. Según creo, dichas estrategias pueden considerarse expresión no de una especie de dualismo entre lo psíquico y lo epistémico, sino más bien de una concepción sumamente fecunda de la racionalidad humana que incorpora la formación de imágenes mentales ricas y complejas como parte estructural de la dinámica argumental humana sin que ello implique la aceptación de ninguna dimensión irracional o afectiva. ${ }^{27}$

Por ejemplo, la descripción que encontramos en De morte del sabio epicúreo como un individuo que camina por la vida "ya enterrado" (entetaphiasménos peripateî; De morte, XXXVIII, 14-19 Kuiper) o la enumeración en XXXII.31-XXXIII.9 de los múltiples lugares en los que podríamos encontrar la muerte (a saber, en el mar, en un pantano o en un río) y del tipo de animales que podrán devorar nuestro cadáver una vez que hayamos muerto (peces, gusanos o larvas) no parecen en modo alguno obligarnos a suponer que Filodemo renuncie a la confianza epicúrea en las capacidades racionales de sus discípulos y lectores y apele a una dimensión que vaya más allá de lo argumental. Por el

figuras de la ekphrasis y la enargeia (desde un punto de vista cronológico, el término enargeia es el primero en utilizarse en forma consistente para designar una técnica descriptiva específica). Si bien G. Zanker postula el concepto de enargeia de Epicuro (y no de Filodemo) como el primer momento en el cual deviene en un término técnico, el punto de contacto que el autor propone entre ese uso y la técnica retórica de "poner ante los ojos" ciertos lugares, situaciones o personas, a través de una descripción vívida, parece, cuanto menos, superficial; cfr. Zanker 1981, pp. 308310. Respecto de la evolución del objeto de la ekphrasis, cfr. Webb 2009, pp. 87106; respecto de la relación entre Filodemo y Virgilio, cfr. los artículos reunidos en Armstrong et al. 2004.

${ }^{27}$ Desde luego, esto no significa que debamos atribuir al epicureísmo una concepción monista del alma como la estoica, lo que quiere decir es que las estrategias retóricas de visualización de Filodemo no nos obligan a incorporar ninguna dimensión no racional en la ecuación. En otras palabras, si en efecto el epicureísmo supuso que existía una parte no racional del alma, ésta no parece haber tenido ningún lugar en el esquema básico de su teoría de la acción. 
contrario, de lo que se trata es de lograr que el lector comprenda, en un sentido profundo, el carácter liberador que se desprende de la conciencia en la inminencia de la muerte, en el primer caso (mediante una hipérbole similar al epigrama tercero), y la igualdad de todas las muertes, en el segundo caso. ${ }^{28}$ ¿En qué sentido trascienden las estrategias descriptivas de Filodemo la mera enunciación de las máximas epicúreas abstractas relativas a la muerte? En el primer caso, a través del énfasis i) en que la idea de que la vida humana es finita se aplica a toda vida humana, ii) en que, en consecuencia, todo momento puede ser el último, y por último, iii) en que la conciencia de este hecho es una marca constitutiva de la sabiduría. En el segundo caso, la trascienden a través de la trivialización de la muerte que se alcanza con la equiparación, casi irreverente, de la muerte en el océano con la muerte en una bañera. Esa equiparación funciona, según señala Tsouna, como una ridiculización de la reacción trágica frente a la muerte en el mar propia de la épica anterior a Filodemo. ${ }^{29}$

Por su parte, De oeconomia y De ira apelan a una cantidad importante de estrategias expositivas con una fuerte carga retórica, desde la hipérbole y la presentación sarcástica de personajes hasta la tradicional contraposición entre exempla positivos y negativos, ${ }^{30}$ estrategias que considero (contra Tsouna 2007, pp. 75-67) que no sólo no requieren, sino que no admiten que se las clasifique en un conjunto diferente al de las estrategias intelectuales. En particular, De oeconomia ofrece dos vías de acceso principales: la primera consiste en la discusión de Filodemo sobre la pertinencia y efectividad de las prescripciones que ofrecen Jenofonte y el Pseudo-Aristóteles en sus obras sobre economía doméstica; la segunda, que es la que me interesa aquí, la representa el sutil contrapunto que Filodemo construye en forma gradual entre, por un lado, la figura del individuo que logra administrar sus bienes materiales de manera virtuosa y, por otro lado, el que permite que su preocupación por la preservación de sus bienes se convierta en una fuente de pertur-

${ }^{28}$ Schroeder remite, curiosamente, a este último pasaje (De morte XXXII.31XXXIII.9) cuando desarrolla la idea de que las técnicas de visualización de Filodemo dan cuerpo a una psicoterapia que, al liberarse de la tiranía del logos, se aleja de las directivas epicúreas ortodoxas; cfr. Schroeder 2004, p. 142.

${ }^{29}$ Cfr. Tsouna 2007, p. 294.

${ }^{30}$ Sobre el marco general en el que se ubican los exempla, en particular en Lucrecio, cfr. Erler 2002, pp. 177-179. Como señala el propio Erler, el recurso a los exempla en el epicureísmo italiano se articula con la actitud más abierta que Filodemo y Lucrecio muestran respecto de la paideia tradicional, la cual les permite incorporar en el catálogo de exempla — tanto positivos como negativos- personajes provenientes de la épica y de la tragedia; cfr. Erler 2011, p. 23.

Diánoia, vol. LXII, no. 79 (noviembre de 2017). 
baciones anímicas. A lo largo del tratado, el autor hilvana la figura casi grotesca del philokremátos como un sujeto cuyo deseo de control lo lleva a "levantarse antes que los sirvientes y acostarse después que ellos" (De oec. XI.30-35), a obsesionarse con cada detalle de sus propiedades materiales (hacienda, riquezas, etc.) (XIX.10-15) y a agonizar por cada pérdida (perí tôn elatt [ōmát]ōn agōniân; XIV.30-35). El objetivo de esta construcción es claro: por un lado, desplegar en forma precisa y concreta las consecuencias cotidianas que se derivan de la valoración de los bienes materiales como fines en sí mismos en lugar de considerarlos meros instrumentos; por otro lado, presentar una lista de "síntomas" ante los cuales el lector debe estar atento si desea evitar considerar en forma indebida el lugar que dichos bienes deben ocupar en el cálculo hedonístico. Nada de esto, como es evidente, pretende apelar a otra cosa que a las capacidades racionales: el objetivo del tratado es que comprendamos qué es lo que no debemos hacer, que podamos comprender la contradicción lógica que existe entre a) la pretensión del philokremátos de que su preocupación por sus bienes materiales responde a un deseo de garantizar su propia felicidad, entendida como ausencia de perturbaciones, $\mathrm{y} b$ ) el hecho de que dicha preocupación (que deviene en celo, obsesión) lo aleja precisamente de ese objetivo.

¿Podemos establecer conclusiones similares para las descripciones que encontramos en De ira (las cuales Annas califica como "tácticas de choque"; Annas 1989, p. 156), o debemos suponer, por el contrario, con Armstrong, que sirven para apelar a las emociones? ${ }^{31}$ Considero que la lectura de De ira 3 evidencia que de lo que se trata es, a todas luces, de una estrategia en esencia racionalista (vinculada, por otra parte, por Filodemo de manera explícita con una concepción cognitivista del alma humana). El objetivo general del tratado no consiste en ofrecer una definición abstracta de la ira, con la cual cualquier discípulo probablemente estaría de acuerdo y creería comprender en forma cabal, sino en exponer, como dicho pasaje afirma, las consecuencias concretas, específicas, que se siguen de tal definición; ${ }^{32}$ en enumerar, en palabras de Filodemo, entre la multiplicidad de males que se siguen de la ira, "aquellos que ignoramos, aquellos que olvidamos, aquellos que no tomamos en consideración adecuadamente (al menos en cuanto a su gravedad), aquellos que comprendemos en toda su complejidad" (De ira 3, col. iii; la traducción es mía). El fin ulterior al que se subordina esta enumeración es que el discípulo pueda evaluar en forma integral, sopesar con

${ }^{31}$ Cfr. Armstrong 2004, p. 26, citado supra p. 150.

${ }^{32}$ Para un intento de reconstrucción de la definición exacta de la ira (definición que no se encuentra en lo que se ha preservado del tratado), cfr. Asmis 2011, p. 165. 
exactitud, "la gravedad de la enfermedad y de los males que nacen

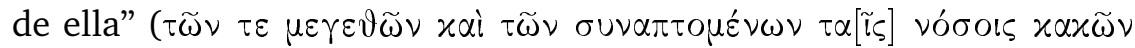

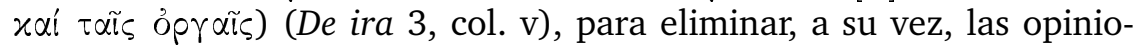
nes falsas del alma que provocan los accesos de ira (De ira 3, col. vi). De manera similar, la descripción (anagraphōn; De ira 3, col. iii) de la sintomatología externa de la ira tiene por objetivo explícito "poner ante los ojos" ( estéticamente desagradable, indigno, de la ira, con el fin de que el discípulo o lector huya de su camino en el momento preciso en que tal descripción le venga a la mente (De ira 3, col. iii). Este recurso, cabe insistir, nada tiene de irracional en la medida en que sólo funciona con éxito cuando lo contrastamos con un ideal determinado de conducta, decoro, etc., un ideal ante el cual el cuadro que esboza Filodemo no puede más que generar rechazo: el cuello tenso, las venas inflamadas, la saliva amarga y salada (De ira 1, fr. 6), la respiración más agitada "que aquellos que han corrido mil estadios" (De ira 4, col. ix). ${ }^{33}$

\section{Conclusiones}

Si, junto con Armstrong y J.R. Irvine, aceptamos que Epicuro desestimó por completo la retórica, ${ }^{34}$ ¿podemos hacer lo mismo que hicimos en relación con las reflexiones teóricas de Filodemo sobre la poesía, esto es, justificar sus investigaciones teóricas sobre la retórica como una investigación no necesaria, pero que permite la normativa epicúrea? Nada lo impide. Más aún: podríamos juntar dicha justificación con la sugerencia de D. Delattre de interpretar la apertura disciplinar de Filodemo (y de su maestro Zenón) como una estrategia esencialmente proselitista. ${ }^{35}$ Desde esa perspectiva combinada, la reflexión teórica tanto sobre

${ }^{33}$ Ciertamente, los casos del individuo que, airado, salta del lecho desnudo y comienza a perseguir, en busca de pelea, a alguien que se le acerca (De ira 4, col. x) y de quienes se revientan un pulmón o experimentan dolores físicos y afecciones que les producen la perspectiva de la muerte (De ira 4, col. ix) parecen hiperbólicos y difíciles de conectar con una situación realista. No obstante, si recordamos que la interpretación alternativa de la ira contra la cual escribe Filodemo es la peripatética, la hipérbole se justifica plenamente en la medida en que pretende reducir al absurdo la justificación aristotélica de la ira como un acicate para la acción.

${ }^{34}$ Cfr. Armstrong 2004, p. 26 e Irvine 1971, p. 96.

35 "L'intelligence rusée d'un Philodème, bon connaisseur de la mentalité des aristocrates romains qu'il fréquentait quotidiennement lui fit-elle développer et amplifier considérablement l'intérêt que son maître Zénon de Sidon avait commencé de porter à ce domaine, dans le but de piquer la curiosité des nouveaux maîtres du monde et, de la sorte, de retenir leur attention, comme malgré eux. Non, écrit-il 
la poesía como sobre la retórica podrían en efecto considerarse una incursión en campos hasta el momento desatendidos por el epicureísmo con fines puramente tácticos, esto es, para construir un terreno común con el auditorio que permitiera a Filodemo atraer a sus discípulos por el camino de la conversión a través de la discusión de temas que interesaran al auditorio y de la conformación de un espacio para dar rienda (relativamente) suelta a ciertos placeres intelectuales. ${ }^{36}$

Dicha interpretación es ciertamente digna de atención y no parece presentar problemas de tipo hermenéutico, al menos en lo que respecta a Filodemo. ${ }^{37}$ Sin embargo, otra interpretación es posible, la cual permite articular la reflexión teórica sobre la retórica que se ha conservado de Filodemo con la dimensión retórica de su producción escrita (sus obras en prosa y sus epigramas): si consideramos que el objetivo que el epicureísmo asigna al discurso filosófico (esotérico) consiste en persuadir al discípulo o lector respecto de la necesidad de guiarse por ciertos principios filosóficos fundamentales (y ésa es, a fin de cuentas, la razón por la cual asigna un lugar absolutamente central a la claridad), la posibilidad de potenciar -mediante el estudio de ciertos aspectos de la técnica retórica - la efectividad persuasiva del discurso filosófico no parece algo que Epicuro podría haber desaconsejado. En este sentido y luego de analizar los límites que Filodemo asigna a un buen uso de ciertas téchnai (más allá de los cuales cada téchne se vuelve innecesaria y perjudicial por ser demasiado técnica, especializada o teorética), D. Blank concluye:

au livre IV de la Musique (col. 140-144 7) avec une fureur qu'il a bien du mal à contenir, les épicuriens ne sont pas des 'rustres', des àrporxó, mais des gens

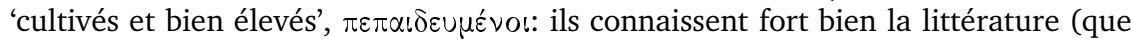
de citations, sans doute fidèles, de grands auteurs dans ce qui nous est parvenu de l'œuvre philosophique du Gadaréen!), la rhétorique, la poésie ou la musique, et savent les apprécier à l'occasion. Et, s'ils se montrent critiques vis-à-vis de ceux qui en usent fort mal à leurs yeux, en leur consacrant l'essentiel de leur activité, c'est uniquement pour des motifs hautement philosophiques, et pas du tout par mépris pour de telles occupations ou, ce qui serait pire, par ignorance" (Delattre 2002, pp. 239-240).

${ }^{36}$ Hubbell sugiere además interpretar las teorizaciones de Filodemo sobre el arte retórica con el trasfondo del resurgimiento de ciertas escuelas isocráticas. Cfr. Hubbell 1916, pp. 410-411.

${ }^{37}$ No obstante, en relación con Epicuro, la imagen radical propuesta por Armstrong (esto es, la imagen de un Epicuro que rechaza por completo el estudio y utilización de la retórica) ha sido descartada casi por todos, sobre todo por la dimensión retórica de los epítomes que se han conservado (epístolas y colecciones de sentencias breves). Cfr., entre otros, Arrighetti 2013. 
La retórica sofística [...], el arte de escribir discursos, ofrecer demostraciones públicas y estructurar los tratados, es un arte formalista con método (aunque no demasiado), tal como sucede con la poesía. Más aún, dado que los filósofos (y otros individuos) deben por lo general hablar o escribir, dicho arte es útil, aunque debe evitar la elaboración excesiva, la cual presumiblemente entraría en conflicto con el objetivo de la claridad, a la vez que requeriría un trabajo arduo que distraería al filósofo de la poesía. El objetivo de la retórica es persuadir a través de un logos retórico, no a través de otros medios, por ejemplo, dialéctica o drásticamente, como hacía Friné cuando utilizaba la belleza y la música como herramientas de persuasión. Ahora bien: ¿cuál es la retórica que hace que el logos sea capaz de persuadir retóricamente? Al parecer, la naturaleza nos ha dado la capacidad de hablar, pero es la téchne la que nos da la capacidad de hablar bien, es decir, con persuasión. (Blank 1996, pp. 594-595) ${ }^{38}$

Si esto es así, en la medida en que la retórica pueda mantenerse dentro de los límites que define la subordinación al objetivo de la persuasión por medios racionales, el estudio de los fundamentos de la persuasión y el recurso a estrategias retóricas como las que resume Sider en el caso de los epigramas o las que expuse en la sección anterior en relación con De morte, De oeconomica y De ira no parecen presentar riesgo alguno desde el punto de vista de la ortodoxia epicúrea.

Como síntesis final, podemos volver al ejemplo de la ira que vimos antes: para Filodemo, la comprensión de que la ira es un estado emocional que deber evitarse (salvo en contadas excepciones) no puede obtenerse de la mera lectura de dicha proposición: el discípulo escuchará hasta el hartazgo máximas sobre el carácter nocivo de la ira y las repetirá en su interior al repasar en su mente o junto con otro discípulo los epítomes epicúreos que circulan en la comunidad, pero ello no será suficiente para que alcance una comprensión profunda de las razones por las cuales la ira debe evitarse, o de las consecuencias que se derivan, desde el punto de vista de la eudaimonía, del hecho de ceder a los ataques de ira. La mera lectura y repetición de máximas abstractas no puede calar lo suficientemente profundo en su alma como para evitar que subsistan en su disposición anímica porciones de territorio no conquistadas por la prédica del maestro (y, dicho sea de paso, es justo sobre esta posibilidad sobre la que se construye el dispositivo de la parrhesía). ${ }^{39}$ Cabe advertir que dichos territorios indómitos no representan reductos de irracionalidad, sino situaciones potenciales, escenarios aún

${ }^{38}$ Cfr. asimismo Blank 2002, p. 249 y Gaines 2004.

${ }^{39}$ Hasta cierto punto, la práctica de la parrhesía parece funcionar sobre un terreno similar al de la teoría estoica de los dos tipos de asentimiento: para el estoi- 
sin visitar, alternativas probables pero que todavía no se han explorado con la razón. En consecuencia, la conquista de esos territorios no dependerá de estrategias retóricas que pongan en juego elementos no racionales, "puramente emocionales" del alma, sino todo lo contrario: la exposición, profunda, detallada, gráficamente exacta de lo que significa, por ejemplo, ser presa de la ira así como de las consecuencias que de ello se siguen. En síntesis, este tipo de estrategias de tipo descriptivo no sólo no pone en riesgo la integridad del proyecto racionalista, sino que, muy por el contrario, deviene en una de las armas más poderosas de las que dispone el maestro en su búsqueda de una estrategia que le permita trascender los límites de un racionalismo que se basa en la lectura y repetición de consignas ciertas pero abstractas.

\section{BIBLIOGRAFÍA}

Obras de Filodemo de Gadara

De Electionibus et Fugis. On Choices and Avoidances, trad. y ed. G. Indelli y V. Tsouna, Bibliopolis, Nápoles, 1997.

De ira. L'ira, ed. G. Indelli, Bibliopolis, Nápoles, 1988.

De Libertate Dicendi. On Frank Criticism, trad. D. Konstan, D. Clay, C.E. Glad, J.C. Thom y J. Ware, Society of Biblical Literature, Atlanta, 2007.

De Oeconomia. On Property Management, trad. V. Tsouna, Society of Biblical Literature, Atlanta, 2012.

De Signis. On Methods of Inference, trad. Ph. De Lacy y E. Allen De Lacy, American Philological Association, Filadelfia, 1941.

Epicurea, ed. H. Usener, Teubner, Leipzig, 1887.

Philodemi volumina rhetorica, ed. S. Sudhaus, Teubner, Leipzig, 1895.

cismo (sobre todo durante su periodo romano), el asentimiento que el individuo puede dar a una proposición puede ser o bien débil o bien fuerte (o "estable"); en el primer caso, el hecho de que otorguemos nuestro asentimiento a una proposición no garantiza en absoluto que nuestras acciones futuras vayan a concordar con lo que expresa esa proposición -algo que, por cierto, no sucederá en el segundo caso- - Esto se puede deber a que no estamos en verdad convencidos de aquello a lo que hemos asentido (aun cuando creamos estarlo) o a que no comprendamos en profundidad las consecuencias que se derivan de lo contenido en un principio. La dinámica de la confesión epicúrea parece funcionar sobre un terreno análogo, a saber, la distancia entre las declaraciones externas del alumno epicúreo y sus propias acciones: a fin de cuentas, lo que el discípulo confiesa es que a pesar de que ha asentido a ciertos principios de vida y ha jurado guiar su conducta con base en ellos, por algún motivo u otro no lo ha logrado. En consecuencia, el objetivo de las prácticas parrhesiásticas consiste en atender ese residuo latente que impide que el individuo pueda plasmar en su vida de forma fehaciente los principios que (de manera sincera) ha adoptado. 
Bibliografía secundaria

Annas, J., 1989, "Epicurean Emotions", Greek, Roman, and Byzantine Studies, vol. 30, no. 2, pp. 145-164.

Armstrong, D., 2002, "Philodème et l'appréciation de l'effet poétique par l'intellect (Poèmes V et PHerc. 1676)", en Auvray-Assayas y Delattre 2002, pp. 297-309.

— 2004, "All Things to All Men: Philodemus' Model of Therapy and the Audience of De Morte", en Fitzgerald, Obbink y Stanfield Holland 2004, pp. 15-54.

- 2008, “'Be Angry and Sin Not': Philodemus versus the Stoics on Natural Bites and Natural Emotions", en J.T. Fitzgerald (comp.), Passions and Moral Progress in Greco-Roman Thought, Routledge, Londres, pp. 79-121.

Armstrong, D., J. Fish, P.A. Johnston y M.B. Skinner (comps.), 2004, Vergil, Philodemus, and the Augustans, University of Texas Press, Austin.

Arrighetti, G., 2013, "Forme della comunicazione in Epicuro", en M. Erler y J.E. Hessler (comps.), Argument und literarische Form in antiker Philosophie: Akten des 3. Kongresses der Gesellschaft für antike Philosophie 2010, De Gruyter, Berlín, pp. 315-337.

Asmis, E., 1995, "Epicurean Poetics", en Obbink 1995, pp. 15-34.

— , 2011, "The Necessity of Anger in Philodemus' On Anger", en Fish y Sanders 2011, pp. 152-182.

Auvray-Assayas, C. y D. Delattre (comps.), 2002, Cicéron et Philodème. La polémique en philosophie, Rue d'Ulm, París.

Blank, D., 1996, "Philodemus on the Technicity of Rhetoric", en Giannantoni y Gigante 1996, vol. 2, pp. 585-596.

_, 2002 , "La philologie comme arme philosophique: la connaissance technique de la rhétorique dans l'épicurisme", en Auvray-Assayas y Delattre 2002, pp. 241-257.

Bobzien, S., 2006, "Moral Responsibility and Moral Development in Epicurus' Philosophy", en Reis 2006, pp. 206-229.

Chandler, C. (ed.), 2006, Philodemus On Rhetoric, Books 1 and 2. Translation and Exegetical Essays, Routledge, Nueva York.

Cooper, J.M., 2012, Pursuits of Wisdom: Six Ways of Life in Ancient Philosophy from Socrates to Plotinus, Princeton University Press, Princeton.

Delattre, D., 2002, "Vers un premier bilan des conceptions esthétiques de l'épicurien Philodème de Gadara", en Auvray-Assayas y Delattre 2002, pp. 237-240.

Erler, M., 1996, "Philologia medicans. La lettura delle opere di Epicuro nella sua scola", en Giannantoni y Gigante 1996, vol. 2, pp. 513-526.

— 2002, "Epicurus as Deus Mortalis. Homoiosis Theoi and Epicurean SelfCultivation", en D. Frede y A. Laks (comps.), Traditions of Theology: Studies in Hellenistic Theology: Its Background and Aftermath, Brill, Leiden, pp. 159180. 
Erler, M., 2011, "Autodidact and Student: On the Relationship of Authority and Autonomy in Epicurus and the Epicurean Tradition", en Fish y Sanders 2011, pp. 9-28.

Fish, J. y K.R. Sanders (comps.), 2011, Epicurus and the Epicurean Tradition, Cambridge University Press, Cambridge.

Fitzgerald, J.T., D. Obbink y G. Stanfield Holland (comps.), 2004, Philodemus and the New Testament World, Brill, Leiden.

Gaines, R.N., 2004, "Cicero, Philodemus, and the Development of Late Hellenistic Rhetorical Theory", en Fitzgerald, Obbink y Stanfield Holland 2004, pp. 197-220.

Giannantoni, G. y M. Gigante (comps.), 1996, Epicureismo greco e romano. Atti del Congresso Internazionale, Napoli, 19-26 maggio 1995, Bibliopolis, Nápoles.

Gigante, M., 2002, "Philodème dans l'histoire de la littérature grecque", en Auvray-Assayas y Delattre 2002, pp. 23-50.

Hubbell, H.M., 1916, "Isocrates and the Epicureans", Classical Philology, vol. 11, no. 4, pp. 405-418.

Irvine, J.R., 1971, "The 'Rhetorica' of Philodemus", Western Speech, vol. 35, no. 2, pp. 96-103.

Janko, R., 1995, "Reconstructing Philodemus' On Poems", en Obbink 1995, pp. 69-96.

Karamanolis, G., 2005, "Two Epigrams of Philodemus and Their Implications for His Literary Method (AP 10.21, 5.107)", Mnemosyne, vol. 58, no. 1, pp. 118-124.

Konstan, D., 2006, "Epicurean 'Passions' and the Good Life", en Reis 2006, pp. 194-205.

López Martínez, M.P., 2003, "La Poética de Filodemo de Gádara: estado de la cuestión”, Ítaca. Quaderns Catalans de Cultura Clàssica, no. 19, pp. 115-126.

Nussbaum, M., 1996, The Therapy of Desire. Theory and Praxis in Hellenistic Ethics, Princeton University Press, Nueva Jersey.

Obbink, D. (comp.), 1995, Philodemus and Poetry: Poetic Theory and Practice in Lucretius, Philodemus, and Horace, Oxford University Press, Nueva York.

_ 1997, "The Mooring of Philosophy. A Review of Philodemus, On Choice and Avoidances, ed. with Commentary by G. Indelli and V. Tsouna-McKirahan", Oxford Studies in Ancient Philosophy, no. 15, pp. 259-282.

Procopé, J.F., 1998, “Epicureans on Anger”, en J. Sihvola y T. Engberg-Pedersen (comps.), The Emotions in Hellenistic Philosophy, Springer Netherlands, Dordrecht, pp. 171-196.

Reis, B. (comp.), 2006, The Virtuous Life in Greek Ethics, Cambridge University Press, Cambridge.

Romaschko, S.A., 1996, "The Communicative Patterns and the Language of the Epicurean Texts", en Giannantoni y Gigante 1996, vol. 1, pp. 261-267.

Schroeder, F.M., 2004, "Philodemus: Avocatio and the Pathos of Distance in Lucretius and Vergil", en Armstrong, Fish, Johnston y Skinner 2004, pp. 139-156. 
Sedley, D., 2003, "Philodemus and the Decentralisation of Philosophy", Cronache Ercolanesi, vol. 33, pp. 31-41.

Sider, D. (ed.), 1997, The Epigrams of Philodemos, Oxford University Press, Nueva York.

, 2004, "How to Commit Philosophy Obliquely: Philodemus' Epigrams in the Light of His Peri Parrhesias", en Fitzgerald, Obbink y Stanfield Holland 2004, pp. 85-101.

Sorabji, R., 2000, Emotion and Peace of Mind. From Stoic Agitation to Christian Temptation. The Gifford Lectures, Cambridge University Press, Cambridge.

Tsouna, V., 2007, The Ethics of Philodemus, Oxford University Press, Oxford.

— , 2009, "Epicurean Therapeutic Strategies", en J. Warren (comp.), The Cambridge Companion to Epicureanism, Cambridge University Press, Cambridge, pp. 249-265.

_ 2011 , "Philodemus, Seneca and Plutarch on Anger", en Fish y Sanders 2011, pp. 183-210.

Webb, R., 2009, Ekphrasis, Imagination and Persuasion in Ancient Rhetorical Theory and Practice, Ashgate, Farnham.

Zanker, G., 1981, "Enargeia in the Ancient Criticism of Poetry", Rheinisches Museum für Philologie, vol. 124, nos. 3-4, pp. 297-311. 